\title{
Perspectivas para el estudio del bienestar: Observatorio de Calidad de Vida y Salud Social
}

\author{
L. F. Beltrán - Guerra1 - J. L. Arellanez - Hernández - \\ E. Romero - Pedraza
}

\begin{abstract}
RESUMEN
En el presente trabajo se presenta la propuesta del Observatorio de Calidad de Vida y Salud Social de la Universidad Veracruzana, que -desde los planteamientos de la ONU contenidos en los Objetivos del Desarrollo Sostenible; en el Programa de las Naciones Unidas para el Desarrollo así como diversos estudios del Desarrollo Humano- utiliza una perspectiva multidimensional para abordar los estudios sobre bienestar, desde, con el fin de sistematizar información que contribuya a la toma de decisiones para incidir en la mejora de las condiciones de bienestar y salud social en México.
\end{abstract}

\begin{abstract}
Present work presents the proposal of the Observatory of Quality of Life and Social Health of the Universidad Veracruzana, which -from the proposals of the UN contained in the Sustainable Development Goals; in the United Nations Development Program as well as various studies of Human Development - uses a multidimensional perspective to address welfare studies, in order to systematize information that contributes to decision making to influence the improvement of the conditions of welfare and social health in Mexico.
\end{abstract}

Palabras Clave: Bienestar, Bienestar Subjetivo, Calidad de Vida, Salud Social, Condiciones de Vida.

Keywords: Wellbeing, Subjective Wellbeing, Quality of life, Social healt, Life conditions.

E l Observatorio de Calidad de Vida y Salud Social, se deriva de la iniciativa propuesta por la Universidad Veracruzana de crear espacios que permitan definir problemáticas, monitorear indicadores y contribuir a los procesos de generación de políticas públicas en el Estado de Veracruz. De igual forma, se suma a la propuesta de la Organización de las Naciones Unidades (ONU), en cuanto al planteamiento de los "Objetivos para el desarrollo sostenible".
Se fundamenta en la necesidad de identificar el nivel de bienestar y calidad de vida que los individuos perciben, tal y como lo viven. Se plantea considerar a la calidad de vida y a la salud social, como indicadores que complementen la información proporcionada por los diversos índices que miden el desarrollo humano, la competitividad social y el progreso para con ello contribuir al replanteamiento de las formas en que se gestiona el desarrollo y bienestar, elementos importantes para la calidad de

1 Universidad Veracruzana. Instituto de Investigaciones Psicológicas. Observatorio de Calidad de Vida y Salud Social. Correo:lebeltran@uv.mx

2 Organización de las Naciones Unidas (2015). Objetivos Desarrollo Sostenible. 17 objetivos para transformar nuestro mundo. Disponible en: https://WWW.un.org/sustainabledevelopment/es/objetivos-de-desarrollo-sostenible/ 
vida y la salud social.

Se apunta a la búsqueda de mejorar las condiciones de vida, desde una perspectiva que considera la complejidad del bienestar, a través de la multidisciplinariedad, para el estudio de un fenómeno con características multidimensionales. Se parte de la idea de que, en la búsqueda de generar mejores condiciones para los individuos de una sociedad, los paradigmas que no consideren la complejidad del bienestar y su multidimensionalidad a través de la multidisciplinariedad, pueden limitar la explicación y la toma de decisiones que incidan de manera favorable en la calidad de vida y en la salud social de las personas.

Sin importar la perspectiva, ya sea la que considera variables objetivas o subjetivas en los diferentes campos del conocimiento, no sería conveniente hablar de desarrollo y progreso en un país, cuando las condiciones de bienestar en una sociedad no presentan cambios significativos. En el caso de México, reflejado con diferentes matices en sus entidades federativas, las condiciones de desarrollo humano en que viven las personas se mantienen a lo largo del tiempo, o en su defecto disminuyen, tal y como se muestra en los resultados del Índice de Desarrollo Humano (IDH) en México en los últimos 10 años.

Las características y necesidades de las entidades federativas en México son diversas, con condiciones de bienestar muy variadas. Es por ello recomendable también realizar ejercicios que permitan, analizar los indicadores de calidad de vida y de salud social objetivos y subjetivos, a nivel estatal y municipal, con la intención de ver qué indicadores macroeconómicos se reflejan de manera directa en las condiciones micro, en la calidad de vida y en el bienestar de cada individuo.
El desarrollo económico tradicional ha tenido menos éxito para desarrollar capacidades en las personas, rubro en el cual es eficiente en términos económicos, pero no tan eficiente en términos sociales ${ }^{4}$. Es por ello que se propone integrar sistemas centrados en la calidad de vida y salud social de la población, más que en la medición de la producción económica, orientadas a generar condiciones de sustentabilidad.

El bienestar puede ser un objeto que permita la delimitación para observar y medir el progreso en las sociedades, en tanto se pueda verificar que los indicadores de bienestar macro, se ven reflejados en el bienestar de la gente.

En el ámbito mundial se está gestionando una propuesta que contempla seguir integrando al estudio del bienestar desde una perspectiva multidimensional, variables inherentes al individuo, particularmente aquellas promovidas por el Programa de las Naciones Unidas para el Desarrollo , a partir del Índice de Desarrollo Humano, el Índice de Competitividad Social, y actualmente, la Medición del Desarrollo Económico y el Progreso Social, este último encabezado por Stiglitz, Sen y Fitoussi. ${ }^{5}$

La tendencia en la medición del bienestar es considerar variables económicas y variables relativas a los derechos sociales, con variables que consideran la forma en que los individuos perciben y construyen su realidad, satisfacen sus necesidades, emiten juicios a partir de ello, y emociones derivadas de tales procesos cognitivos y afectivos, integrados en el concepto de Bienestar Subjetivo. ${ }^{6}$

Los indicadores macroeconómicos ya no son suficientes y se requiere también obtener información cuantitativa y cualitativa que permita recuperar la visión de los destinatarios, de la gente que se beneficia con estas acciones,

3 Programa de las Naciones Unidas para el Desarrollo (2012). El Índice de Desarrollo Humano en México: Cambios metodológicos e información para las entidades federativas. Disponible en: http://wWw.undp.org.mx/spip.php?page=publicacion\&id_article $=2338$

4 Sen, A. (1983). Development: Which way now? Economic Journal, Vol. 93. Issue 372. Pp. 745-762. Disponible en: http://links.jstor.org/sici?sici=0013-0133\%28198312\%2993\%3A372\%3C745\%3ADWWN\%3E2.0.CO\%3B2-Y

5 Sen, A., Stiglitz, J., Fitoussi, JP. (2009) Informe de la Comisión para la Medición del Desempeño Económico y Progreso Social. Disponible en: http://wWW.stiglitz-sen-fitoussi.fr/documents/rapport_anglais.pdf

6 Rojas, Mariano (2011). Más allá del ingreso: progreso y bienestar subjetivo. En Rojas, Mariano (Coord.). (2011). La medición del progreso y del bienestar. Propuesta desde América Latina. México: Foro Consultivo, Científico y Tecnológico A.C.. ISBN: 978-607-95050-6-6 Disponible en: http://wWw.foroconsultivo.org.mx/libros_editados/midiendo_el_progreso_2011_esp.pdf 
con la intensión de relacionar los resultados con la realidad de la gente.

Bajo esta perspectiva, focalizar los estudios de bienestar y calidad de vida, resulta de gran importancia, pues los estudios de bienestar han empezado a establecer relaciones con aspectos de salud, particularmente con conductas de riesgo, tales como el consumo de drogas, donde se ha encontrado que la percepción subjetiva de bienestar es menor en jóvenes que han consumido drogas, que entre aquellos que no lo han hecho ${ }^{7}$, así como también relaciones con estudios de percepción de inseguridad y violencia, desempleo, educación, entre otros, que también traen consigo afectaciones a los estilos de vida y por ende a la calidad de vida.

7 Córdova-Alcaráz, Alberto Javier; Rodríguez-Kuri, Solveig Eréndira; Díaz-Negrete, David Bruno Bienestar subjetivo en jóvenes mexicanos usuarios y no usuarios de drogas. Revista Intercontinental de Psicología y Educación, vol. 12, núm. 2, julio-diciembre, 2010, pp. 147-162 Universidad Intercontinental. Distrito Federal, México. Disponible en: http://www. redalyc.org/articulo.oa?id $=80218376008$ 